\title{
Relationship between hemoglobin glycation index and extent of coronary heart disease in individuals with type 2 diabetes mellitus: A cross-sectional study
}

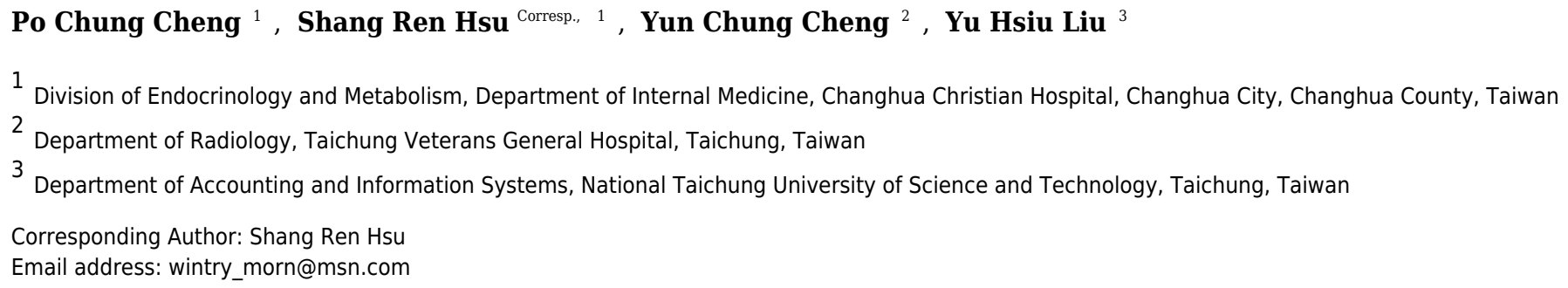

Background. Individuals with type 2 diabetes (T2D) are at an increased risk of coronary heart disease (CHD). Diabetic complications have recently been associated with a measure of glucose metabolism known as the hemoglobin glycation index (HGI). Currently there is insufficient information regarding a potential link between $\mathrm{HGI}$ and cardiovascular disease. This study aimed to investigate the relationship between $\mathrm{HGI}$ and extent of $\mathrm{CHD}$ in individuals with T2D. Methods. This cross-sectional study screened individuals visiting the endocrinology clinic between June 2012 and May 2016 for eligibility. Enrollment criteria included individuals above 21 years of age with T2D diagnosed in the preceding ten years. Candidates with hemoglobin disorders, pregnancy, and existing coronary artery disease were excluded. Fasting plasma glucose (FPG) and glycated hemoglobin Alc ( $\mathrm{HbAlc}$ ) were sampled three months prior to angiography. The regression equation of predicted $\mathrm{HbAlC}=$ 0.008 x FPG +6.28 described the linear relationship between these variables. HGI was calculated as the difference between the measured $\mathrm{HbAlc}$ and predicted $\mathrm{HbAlc}$. Participants were classified into two groups according to the presence of supranormal ( $\geq$ 0 ) or subnormal HGI (<0). Results. Among 423 participants, people with supranormal HGI harbored an increased prevalence of multiple vessel disease relative to those with subnormal HGI (Odds ratio (OR): 3.9, 95\% confidence interval $(\mathrm{Cl}): 2.64-5.98, P<0.001$ ). Moreover, individuals with supranormal HGI more frequently demonstrated lesions involving the left anterior descending artery (OR: 3.0, 95\% Cl: $1.97-4.66, P<0.001$ ). The intergroup difference in mean $\mathrm{HbAlc}$ was statistically nonsignificant $(7.5 \pm 1.0 \%$ versus $7.4 \pm 1.1 \%, P=0.80)$. Discussion. This study demonstrated that HGI correlated with the extent of CHD in individuals with T2D. People with supranormal HGI harbored a higher prevalence of extensive cardiovascular disease compared to those with subnormal HGI. The relationship between $\mathrm{HGI}$ and extent of $\mathrm{CHD}$ enables cardiovascular risk stratification 
in at risk individuals. Overall, HGI provides useful information concerning cardiovascular risk in clinical practice. 
2 Relationship between hemoglobin glycation index and extent of coronary heart disease in individuals with type 2 diabetes mellitus: A cross-sectional study

$5 \quad{ }^{1}$ Division of Endocrinology and Metabolism, Department of Internal Medicine, Changhua

6 Christian Hospital, 135 Nanxiao St., Changhua City, Changhua County, Taiwan

$7 \quad{ }^{2}$ Department of Radiology, Taichung Veterans General Hospital, 1650 Taiwan Boulevard Section

8 4, Taichung, Taiwan

$9{ }^{3}$ Department of Accounting and Information Systems, National Taichung University of Science

10 and Technology, 129 San Min Rd., Taichung, Taiwan

$11{ }^{\dagger}$ Equal contribution to the study

12 Corresponding author: Shang-Ren Hsu

13 Email address: wintry_morn@msn.com

14 Abstract 
15 Background. Individuals with type 2 diabetes (T2D) are at an increased risk of coronary heart

16 disease (CHD). Diabetic complications have recently been associated with a measure of glucose

17 metabolism known as the hemoglobin glycation index (HGI). Currently there is insufficient

18 information regarding a potential link between HGI and cardiovascular disease. This study aimed

19 to investigate the relationship between HGI and extent of CHD in individuals with T2D.

20 Methods. This cross-sectional study screened individuals visiting the endocrinology clinic

21 between June 2012 and May 2016 for eligibility. Enrollment criteria included individuals above

2221 years of age with T2D diagnosed in the preceding ten years. Candidates with hemoglobin

23 disorders, pregnancy, and existing coronary artery disease were excluded. Fasting plasma glucose

24 (FPG) and glycated hemoglobin A1c (HbA1c) were sampled three months prior to angiography.

25 The regression equation of predicted $\mathrm{HbA} 1 \mathrm{c}=0.008 \times \mathrm{FPG}+6.28$ described the linear

26 relationship between these variables. HGI was calculated as the difference between the measured

$27 \mathrm{HbA1c}$ and predicted HbA1c. Participants were classified into two groups according to the

28 presence of supranormal $(\geq 0)$ or subnormal HGI $(<0)$.

29 Results. Among 423 participants, people with supranormal HGI harbored an increased

30 prevalence of multiple vessel disease relative to those with subnormal HGI (Odds ratio (OR): 3.9,

$3195 \%$ confidence interval $(\mathrm{CI}): 2.64-5.98, P<0.001)$. Moreover, individuals with supranormal

32 HGI more frequently demonstrated lesions involving the left anterior descending artery (OR: 3.0,

$3395 \% \mathrm{CI}: 1.97-4.66, P<0.001)$. The intergroup difference in mean HbA1c was statistically

34 nonsignificant $(7.5 \pm 1.0 \%$ versus $7.4 \pm 1.1 \%, P=0.80)$.

35 Discussion. This study demonstrated that HGI correlated with the extent of CHD in individuals

36 with T2D. People with supranormal HGI harbored a higher prevalence of extensive

37 cardiovascular disease compared to those with subnormal HGI. The relationship between HGI

38 and extent of CHD enables cardiovascular risk stratification in at risk individuals. Overall, HGI 
39 provides useful information concerning cardiovascular risk in clinical practice.

40 Relationship between hemoglobin glycation index and extent of coronary heart disease in

421 Introduction 
43 Type 2 diabetes mellitus (T2D) is a developing epidemic that affects a substantial proportion of

44 the adult population (Chen, Magliano \& Zimmet, 2011). Changes in dietary habit, urbanization,

45 and sedentary lifestyle contribute to an increasing incidence of the disease (Yang et al., 2010).

46 Hyperglycemia exerts detrimental effects on blood vessels, as evidenced by a predisposition to

47 develop retinopathy, nephropathy, and coronary heart disease (CHD) (Mohammedi et al., 2017).

48 These vascular complications profoundly influence the quality of life in affected individuals.

49 Specifically, individuals with T2D are at risk of developing cardiovascular disease (Shah et al.,

50 2015), which accounts for nearly sixty percent of diabetes related mortality (Kalofoutis et al.,

51 2007). Although glycemic control as represented by glycated hemoglobin A1c (HbA1c)

52 influences vascular disease, this association is not particularly robust (Laakso, 2010).

53 Investigators have proposed that elements of hyperglycemia not captured by HbA1c

54 measurement may modify cardiovascular risk (Fox, 2010).

55 The hemoglobin glycation index (HGI) is an indicator of glucose metabolism linked to diabetic

56 complications (Soros et al., 2010). HGI correlated with a composite index of cardiac, cerebral,

57 and peripheral vascular events in a recent study involving individuals with T2D (Nayak, 2013).

58 Specifically, this glycation index may correlate with cardiovascular disease risk. This study

59 investigated the relationship between HGI and extent of coronary vascular disease in people with

60 T2D.

612 Materials and Methods

62 2.1 Study population 
63 This cross-sectional study screened patients visiting the endocrinology clinic at Changhua

64 Christian Hospital, Changhua City, Taiwan, between June 2012 and May 2016 for eligibility.

65 Enrollment criteria included individuals exceeding 21 years of age, with T2D diagnosed in the

66 preceding ten years, who received hydroxymethylglutaryl-coenzyme A (HMG-CoA) reductase

67 inhibitors since diabetes onset and underwent coronary angiography (CAG) during the study

68 period. Exclusion criteria involved people who had undertaken CAG prior to the study, those

69 with existing CHD, or who lacked concomitant HbA1c and fasting plasma glucose (FPG)

70 measurements. Candidates with hemoglobin disorders, pregnancy, and congenital coronary artery

71 abnormalities were also ineligible. Decision to perform coronary artery survey was made by

72 cardiologists based on high risk findings on non-invasive testing or high pre-test probability of

73 coronary artery disease. All participants provided written informed consent for CAG. The study

74 was approved by the Institutional Review Board of Changhua Christian Hospital (CCH IRB

75 number: 161111).

76 2.2 Calculation of HGI

77 Concomitant HbA1c and FPG measurements from all participants were graphed to establish the

78 linear relationship between these variables, as shown in Figure 1, from which the regression

79 equation of predicted $\mathrm{HbAlc}=0.008 \times \mathrm{FPG}+6.28$ was derived. Individual FPG in milligrams

80 per deciliter was substituted into this linear regression equation to derive the predicted HbA1c.

81 HGI was calculated as the difference between the measured HbA1c and predicted HbAlc

82 (Hempe et al., 2015). Supranormal HGI was defined as levels above or equivalent to zero,

83 whereas subnormal HGI designated values below zero. 
842.3 Classification of CHD

85 The extent of vascular disease involving the left anterior descending, left circumflex, and right

86 coronary arteries was documented by CAG. Significant stenosis was defined as more than fifty

87 percent narrowing of the diseased vascular segment compared to a proximal or distal normal

88 segment (Leopold \& Faxon, 2015). Single vessel disease was defined as one or more stenotic

89 lesions in one of the major coronary arteries, whereas multiple vessel disease involved lesions in

90 two or more of the coronary arteries (Tazaki et al., 2013). Arteriosclerosis described the

91 observation that none of the stenotic lesions resulted in more than fifty percent narrowing of the

92 major coronary arteries.

$93 \quad 2.4$ Statistical analysis

94 Baseline characteristics including age, gender, lipid profile, mean $\mathrm{HbAlc}$, and cigarette smoking

95 were compared between the HGI subgroups. Intergroup comparisons were made using Student's

$96 t$ test for continuous variables and Pearson's $\chi^{2}$ test for categorical variables. For the HGI

97 subgroups, the prevalence of multiple vessel disease as opposed to single vessel disease or

98 arteriosclerosis was compared using Pearson's $\chi^{2}$ test. Tests of statistical significance were based

99 on a two-tailed $P<0.05$. Statistical analysis was performed using Statistical Package for the

100 Social Sciences (version 22.0, SPSS, Chicago, IL). A multivariate regression analysis was

101 performed to evaluate the contribution of HGI and other risk factors to CHD. A logistic

102 regression analysis adjusted for confounders demonstrated the association between the HGI

103 subgroups and extent of cardiovascular disease.

1043 Results 
105 Initially 480 individuals with T2D were screened for eligibility. Twenty patients were excluded

106 due to lack of HMG-CoA reductase inhibitor prescription, 32 individuals had received CAG prior

107 to the study and were ineligible, and five candidates were excluded due to absence of

108 concomitantly measured HbA1c and FPG. Figure 2 illustrates the enrollment process of this

109 study.

110 The study enrolled 423 individuals who were classified according to the presence of either

111 supranormal $(\geq 0)$ or subnormal HGI $(<0)$. Baseline characteristics including age, gender, and

112 kidney function were similar between groups, as summarized in Table 1. Levels of low-density

113 lipoprotein cholesterol, presence of cigarette smoking, and degree of systolic blood pressure were

114 also comparable. The intergroup difference in mean HbAlc was nonsignificant $(7.5 \pm 1.0 \%$

115 versus $7.4 \pm 1.1 \%, P=0.80)$, and both HGI groups harbored similar degree of proteinuria $(0.25 \pm$

1160.95 grams per day versus $0.19 \pm 0.57$ grams per day, $P=0.467$ ).

117 As shown in Table 2, individuals with supranormal HGI harbored a higher prevalence of multiple

118 vessel disease relative to those with subnormal HGI (Odds ratio (OR): $3.9,95 \%$ confidence

119 interval (CI): $2.64-5.98, P<0.001)$. Moreover, the supranormal HGI group more frequently

120 demonstrated lesions involving the left anterior descending artery (OR: 3.0, 95\% CI: 1.97 - 4.66,

$121 P<0.001)$. As illustrated in Table 3, the length of hospitalization was similar between groups.

122 Intriguingly, people with supranormal HGI demonstrated a trend towards requiring multiple stent 123 deployment relative to those with subnormal HGI $(23.0 \%$ versus $16.7 \%, P=0.067)$.

124 A multivariate regression analysis was performed using age, systolic blood pressure, low density 125 and high density lipoprotein cholesterol, triglycerides, and HGI to determine the effect of these 
126 variables on CHD. The standardized coefficient of each variable in association with

127 cardiovascular disease is shown in Table 4, which identifies HGI as a prominent contributor to

128 the extent of CHD $(\beta=0.31 ; P<0.001)$.

129 A logistic regression analysis also demonstrated the association between HGI and extent of CHD.

130 After adjusting for potential confounding variables, participants with supranormal HGI

131 nonetheless harbored a higher risk of multiple vessel disease (OR: 2.62, 95\% CI: 1.97-3.49, $P<$

$1320.001)$ compared with those with subnormal HGI.

1334 Discussion

134 Cardiovascular disease affects a considerable proportion of people with T2D and detracts from

135 their survival (Naito\& Kasai, 2015; White et al., 2016). However, HbA1c measurements

136 delineated only a fraction of cardiovascular disease risk (Rawshani et al., 2017). This study

137 demonstrated that HGI consistently correlated with the extent of CHD in T2D. As observed by

138 previous investigators, people with elevated HGI harbor an accelerated rate of protein glycation

139 with subsequent endothelial injury (Nayak et al., 2011). Supranormal HGI may also reflect an

140 excess of advanced glycosylation end products that arise from chronic hyperglycemia (Singh et

141 al., 2014).

142 Researchers have postulated that specific patterns of glucose variation, as measured by HGI,

143 constitute a distinctive risk factor for diabetic complications. Whereas HbA1c measures protein

144 glycosylation within the red blood cell, diabetic complications may arise from protein glycation

145 in both the extracellular and intracellular space. HGI was suggested as an indicator of the rate of

146 extracellular protein glycation (Leslie \& Cohen, 2009). Another potential explanation for a 
147 supranormal HGI may be postprandial hyperglycemia, which raises the measured HbAlc above

148 that predicted from FPG (Rizza, 2010; Riddle \& Gerstein, 2015). Indeed, several studies have

149 implicated hyperglycemia after meals in the development of cardiovascular disease (Cavalot et

150 al., 2011; Node \& Inoue, 2009; Ceriello, 2009). These findings were corroborated by the

151 observation in this study that different HGI subgroups, potentially reflecting the degree of

152 postprandial hyperglycemia, consistently correlated with the extent of CHD.

153 Whereas researchers previously identified an association between HGI and a composite index of

154 cardiac, cerebral, and peripheral vascular events (Nayak et al., 2013; Hempe et al., 2015), this

155 study provides novel information by focusing on the link between HGI and coronary vascular

156 disease. An implication of this study is that cardiovascular risk assessment may be refined by

157 HGI, particularly in individuals with similar baseline HbA1c. Furthermore, people with

158 supranormal HGI who experience angina pectoris are at risk of CHD and should receive

159 comprehensive examination. Considering the aforementioned link between postprandial

160 hyperglycemia, HGI, and cardiovascular disease risk (Riddle \& Gerstein, 2015; Raz et al., 2011),

161 controlling postprandial hyperglycemia in individuals with supranormal HGI may be an

162 appropriate therapeutic approach to attenuate CHD.

163 This study benefits from an objective assessment of macrovascular disease by CAG.

164 Conventional risk factors for CHD were similar between the HGI subgroups. Furthermore,

165 potential confounding effects of lipid-lowering therapy were reduced by enrolling recipients of

166 HMG-CoA reductase inhibitors since diabetes outset (Jellinger et al., 2012). Data regarding

167 cardiovascular risk factors such as dyslipidemia, blood pressure, and cigarette smoking were

168 available for the entire study population. 
169 Several limitations may arise from the study design. Degree of insulin resistance and mode of

170 antidiabetic treatment may influence CHD but were not uniformly available (Syed Ikmal et al.,

171 2013; Marso et al., 2016). Although participants received comprehensive diabetes education by

172 certified educators, adherence to lifestyle intervention could not be ascertained. Body weight may

173 influence cardiovascular risk, but investigators previously demonstrated that high-risk coronary

174 anatomy was paradoxically less frequent in obese patients (Rubinshtein et al., 2006). The

175 relationship between body weight and CHD remains uncertain and was not assessed in this study.

176 Participants were diagnosed with T2D in the preceding ten years according to their medical

177 records, but precise disease duration could not be confirmed due to the latent nature of T2D.

178 Finally, any complication associated with HGI may be difficult to dissect from the influence of

179 HbA1c (Sacks, Nathan \& Lachin, 2011).

1805 Conclusions

181 HGI consistently correlated with the extent of CHD in individuals with T2D. People with

182 supranormal HGI harbored a higher prevalence of multiple vessel disease compared to those with

183 subnormal HGI, which may further complicate their management. Undeniably, a correlation

184 between HGI and CHD does not imply causation, and further studies are necessary to explore the

185 physiologic basis of this glycation index. Overall, HGI provides useful information regarding

186 cardiovascular disease risk in T2D.

187 References

188 Chen L, Magliano DJ, Zimmet PZ. 2011. The worldwide epidemiology of type 2 diabetes 
189

190

191

192

193

194

195

196

197

198

199

200

201

202

203

204

205

206

207

208

209

210

211

212

213

mellitus-present and future perspectives. Nat Rev Endocrinol 8:228-236.

Yang W, Lu J, Weng J, Jia W, Ji L, Xiao J, Shan Z, Liu J, Tian H, Ji Q, Zhu D, Ge J, Lin L,

Chen L, Guo X, Zhao Z, Li Q, Zhou Z, Shan G, He J; China National Diabetes and

Metabolic Disorders Study Group. 2010. Prevalence of diabetes among men and women in China. N Engl J Med 362:1090-1101.

Mohammedi K, Woodward M, Marre M, Colagiuri S, Cooper M, Harrap S, Mancia G, Poulter N, Williams B, Zoungas S, Chalmers J. 2017. Comparative effects of microvascular and macrovascular disease on the risk of major outcomes in patients with type 2 diabetes.

Cardiovasc Diabetol 16:95.

Shah AD, Langenberg C, Rapsomaniki E, Denaxas S, Pujades-Rodriguez M, Gale CP, Deanfield J, Smeeth L, Timmis A, Hemingway H. 2015. Type 2 diabetes and incidence of cardiovascular diseases: a cohort study in 1.9 million people. Lancet Diabetes Endocrinol 3:105113.

Kalofoutis C, Piperi C, Kalofoutis A, Harris F, Phoenix D, Singh J. 2007. Type II diabetes mellitus and cardiovascular risk factors: Current therapeutic approaches. Exp Clin Cardiol 12:1728.

Laakso M. 2010. Cardiovascular disease in type 2 diabetes from population to man to mechanisms: the Kelly West Award Lecture 2008. Diabetes Care 33:442-449.

Fox CS. 2010. Cardiovascular Disease Risk Factors, Type 2 Diabetes Mellitus, and the Framingham Heart Study. Trends Cardiovasc Med 20:90-95.

Soros AA, Chalew SA, McCarter RJ, Shepard R, Hempe JM. 2010. Hemoglobin glycation index: a robust measure of hemoglobin A1c bias in pediatric type 1 diabetes patients. Pediatr Diabetes 11:455-461.

Nayak AU, Nevill AM, Bassett P, Singh BM. 2013. Association of glycation gap with mortality and vascular complications in diabetes. Diabetes Care 36:3247-3253. 
214 Hempe JM, Liu S, Myers L, McCarter RJ, Buse JB, Fonseca V. 2015. The hemoglobin

215 glycation index identifies subpopulations with harms or benefits from intensive treatment in the 216 ACCORD trial. Diabetes Care 38:1067-1074.

217 Leopold JA, Faxon DP. 2015. Diagnostic cardiac catheterization and coronary angiography. In;

218 Kasper D, Fauci A, Hauser S, Longo D, Jameson J, Loscalzo J, ed. Harrison's Principles of

219 Internal Medicine, 19th edition. New York: McGraw-Hill.

220 Tazaki J, Shiomi H, Morimoto T, Imai M, Yamaji K, Sakata R, Okabayashi H, Hanyu M,

221 Shimamoto M, Nishiwaki N, Komiya T, Kimura T; CREDO-Kyoto PCI/CABG registry

222 cohort-2 investigators. 2013. Three-year outcome after percutaneous coronary intervention and

223 coronary artery bypass grafting in patients with triple-vessel coronary artery disease: observations

224 from the CREDO-Kyoto PCI/CABG registry cohort-2. EuroIntervention 9:437-445.

225 Naito R, Kasai T. 2015. Coronary artery disease in type 2 diabetes mellitus: Recent treatment

226 strategies and future perspectives. World J Cardiol. 7:119-124.

227 White WB, Kupfer S, Zannad F, Mehta CR, Wilson CA, Lei L, Bakris GL, Nissen SE, 228 Cushman WC, Heller SR, Bergenstal RM, Fleck PR, Cannon CP; EXAMINE Investigators.

229 2016. Cardiovascular mortality in patients with type 2 diabetes and recent acute coronary

230 syndromes from the EXAMINE trial. Diabetes Care 39:1267-1273.

231 Rawshani A, Rawshani A, Franzén S, Eliasson B, Svensson AM, Miftaraj M, McGuire DK,

232 Sattar N, Rosengren A, Gudbjörnsdottir S. 2017. Mortality and cardiovascular disease in type

2331 and type 2 diabetes. $N$ Engl J Med 376:1407-1418.

234 Nayak AU, Holland MR, Macdonald DR, Nevill A, Singh BM. 2011. Evidence for consistency

235 of the glycation gap in diabetes. Diabetes Care 34:1712-1716.

236 Singh VP, Bali A, Singh N, Jaggi AS. 2014. Advanced glycation end products and diabetic

237 complications. Korean J Physiol Pharmacol 18:1-14.

238 Leslie RD, Cohen RM. 2009. Biologic variability in plasma glucose, hemoglobin A1c, and 
239 advanced glycation end products associated with diabetes complications. J Diabetes Sci Technol

$240 \quad 3: 635-643$.

241 Rizza RA. 2010. Pathogenesis of fasting and postprandial hyperglycemia in type 2 diabetes:

242 implications for therapy. Diabetes 59: 2697-2707.

243 Riddle MC, Gerstein HC. 2015. Comment on Hempe et al. The hemoglobin glycation index

244 identifies subpopulations with harms or benefits from intensive treatment in the ACCORD trial.

245 Diabetes Care 38: e170-171.

246 Cavalot F, Pagliarino A, Valle M, Di Martino L, Bonomo K, Massucco P, Anfossi G, Trovati

247 M. 2011. Postprandial blood glucose predicts cardiovascular events and all-cause mortality in

248 type 2 diabetes in a 14-year follow-up: lessons from the San Luigi Gonzaga Diabetes Study.

249 Diabetes Care 34:2237-2243.

250 Node K, Inoue T. 2009. Postprandial hyperglycemia as an etiological factor in vascular failure.

251 Cardiovasc Diabetol 8:23.

252 Ceriello A. 2009. Postprandial hyperglycemia and cardiovascular disease:

253 Is the HEART2D study the answer? Diabetes Care 32:521-522.

254 Raz I, Ceriello A, Wilson PW, Battioui C, Su EW, Kerr L, Jones CA, Milicevic Z, Jacober

255 SJ. 2011. Post hoc subgroup analysis of the HEART2D trial demonstrates lower cardiovascular

256 risk in older patients targeting postprandial versus fasting/premeal glycemia. Diabetes Care

$257 \quad 34: 1511-1513$.

258 Jellinger PS, Smith DA, Mehta AE, Ganda O, Handelsman Y, Rodbard HW, Shepherd MD,

259 Seibel JA; AACE Task Force for Management of Dyslipidemia and Prevention of

260 Atherosclerosis. 2012. American Association of Clinical Endocrinologists' Guidelines for

261 Management of Dyslipidemia and Prevention of Atherosclerosis. Endocr Pract 18:1-78.

262 Syed Ikmal SI, Zaman Huri H, Vethakkan SR, Wan Ahmad WA. 2013. Potential biomarkers

263 of insulin resistance and atherosclerosis in type 2 diabetes mellitus patients with coronary artery 
264 disease. Int J Endocrinol doi: 10.1155/2013/698567.

265 Marso SP, Daniels GH, Brown-Frandsen K, Kristensen P, Mann JF, Nauck MA, Nissen SE, 266 Pocock S, Poulter NR, Ravn LS, Steinberg WM, Stockner M, Zinman B, Bergenstal RM, 267 Buse JB; LEADER Steering Committee; LEADER Trial Investigators. 2016. Liraglutide and 268 cardiovascular outcomes in type 2 diabetes. $N$ Engl J Med 375:311-322.

269 Rubinshtein R, Halon DA, Jaffe R, Shahla J, Lewis BS. 2006. Relation between obesity and 270 severity of coronary artery disease in patients undergoing coronary angiography. Am J Cardiol $27197: 1277-1280$.

272 Sacks DB, Nathan DM, Lachin JM. 2011. Gaps in the glycation gap hypothesis. Clin Chem $273 \quad 57: 150-152$. 


\section{Table $\mathbf{1}$ (on next page)}

Demographic characteristics of the hemoglobin glycation index groups

Data are expressed as mean with standard deviation for continuous variables and number (\%) for categorical variables. Hemoglobin glycation index is defined as the difference between an individual's observed $\mathrm{HbAlc}$ and the $\mathrm{HbAlc}$ predicted from fasting plasma glucose. HbAlc, glycated hemoglobin Alc; HDL-C, high-density lipoprotein cholesterol; LDLC, low-density lipoprotein cholesterol; TG, triglycerides; SCr, serum creatinine; HGI, hemoglobin glycation index. 
1 Demographic characteristics of the hemoglobin glycation index groups 2 3

\begin{tabular}{lccc}
\hline & $\begin{array}{c}\text { HGI }<0 \\
(n=180)\end{array}$ & $\begin{array}{c}\text { HGI } \geq 0 \\
(n=243)\end{array}$ & $P$ value \\
\hline HbA1c (\%) & $7.5 \pm 1.0$ & $7.4 \pm 1.1$ & 0.80 \\
Age (years) & $67 \pm 11$ & $67 \pm 10$ & 0.56 \\
Gender & & & \\
$\quad$ Female & $84(46.7 \%)$ & $98(40.3 \%)$ & 0.19 \\
$\quad 96(53.3 \%)$ & $145(59.7 \%)$ & 0.06 \\
MDL-C (mg/dL) & $42 \pm 12$ & $39 \pm 14$ & 0.38 \\
LDL-C (mg/dL) & $88 \pm 30$ & $91 \pm 34$ & 0.76 \\
TG (mg/dL) & $159 \pm 126$ & $155 \pm 109$ & 0.52 \\
SCr (mg/dL) & $1.5 \pm 1.7$ & $1.6 \pm 1.7$ & 0.79 \\
Cigarette smoking & & & \\
$\quad$ Yes & $138(76.7 \%)$ & $189(77.8 \%)$ & 0.65 \\
$\quad$ No & $42(23.3 \%)$ & $54(22.2 \%)$ & \\
Systolic blood & $136 \pm 24$ & $135 \pm 19$ & 0.47 \\
pressure (mm Hg) & & & $0.19 \pm 0.57$ \\
Proteinuria (g/day) & $0.25 \pm 0.95$ & & \\
\hline
\end{tabular}




\section{Table 2 (on next page)}

Extent of coronary heart disease in hemoglobin glycation index groups

Data are expressed as number (\%) for categorical variables. Hemoglobin glycation index is defined as the difference between an individual's observed $\mathrm{HbAlc}$ and the $\mathrm{HbAlc}$ predicted from fasting plasma glucose. $\mathrm{HGI}$, hemoglobin glycation index; LAD, left anterior descending artery; $\mathrm{Cl}$, confidence interval. 
Extent of coronary heart disease in hemoglobin glycation index groups

\begin{tabular}{clllll}
\hline & $\begin{array}{c}\text { HGI }<0 \\
(n=180)\end{array}$ & $\begin{array}{c}\text { HGI } \geq 0 \\
(n=243)\end{array}$ & $\begin{array}{c}\text { Odds } \\
\text { ratio }\end{array}$ & $95 \%$ CI & $P$ value \\
\hline $\begin{array}{c}\text { Arteriosclerosis } \\
\text { or single vessel } \\
\text { disease }\end{array}$ & $111(61.7 \%)$ & $70(28.8 \%)$ & 3.9 & $2.64-5.98$ & $<0.001$ \\
$\begin{array}{c}\text { Multiple vessel } \\
\text { disease }\end{array}$ & $69(38.3 \%)$ & $173(71.2 \%)$ & & \\
\\
$\begin{array}{c}\text { Left anterior } \\
\text { descending } \\
\text { artery disease }\end{array}$ & $102(56.7 \%)$ & $194(79.8 \%)$ & 3.0 & $1.97-4.66$ & $<0.001$ \\
\hline
\end{tabular}

1

2

3 


\section{Table 3(on next page)}

Clinical outcome of the hemoglobin glycation index groups

Data are expressed as mean with standard deviation for continuous variables and number (\%) for categorical variables. Hemoglobin glycation index is defined as the difference between an individual's observed $\mathrm{HbAlc}$ and the $\mathrm{HbAlc}$ predicted from fasting plasma glucose. $\mathrm{HGl}$, hemoglobin glycation index. 
1 Clinical outcome of the hemoglobin glycation index groups

2

3

\begin{tabular}{lccc}
\hline & $\begin{array}{c}\text { HGI }<0 \\
(n=180)\end{array}$ & $\begin{array}{c}\text { HGI } \geq 0 \\
(n=243)\end{array}$ & $P$ value \\
\hline $\begin{array}{l}\text { Length of stay (days) } \\
\text { Number of stent }\end{array}$ & $2.8 \pm 2.3$ & $3.0 \pm 2.5$ & 0.41 \\
$\quad$ None or single & $150(83.3 \%)$ & $187(77.0 \%)$ & 0.067 \\
$\quad$ Multiple & $30(16.7 \%)$ & $56(23.0 \%)$ & \\
\hline
\end{tabular}




\section{Table 4 (on next page)}

Multivariate regression analysis evaluating coronary heart disease as dependent variable 
1 Multivariate regression analysis evaluating coronary heart disease as dependent variable

2

3

\begin{tabular}{lcc}
\hline \multicolumn{1}{c}{ Independent variables } & $\beta$ coefficient & $P$ value \\
\hline Age & 0.054 & 0.14 \\
Systolic blood pressure & -0.058 & 0.12 \\
Low density lipoprotein & 0.051 & 0.15 \\
cholesterol & & \\
High density lipoprotein & -0.06 & 0.11 \\
cholesterol & & \\
Triglycerides & -0.084 & 0.041 \\
Hemoglobin glycation & 0.31 & $<0.001$ \\
index & & \\
\hline
\end{tabular}


Figure 1

Relationship between hemoglobin Alc and fasting plasma glucose

Linear regression for the calculation of predicted hemoglobin Alc from measured hemoglobin Alc and fasting plasma glucose.

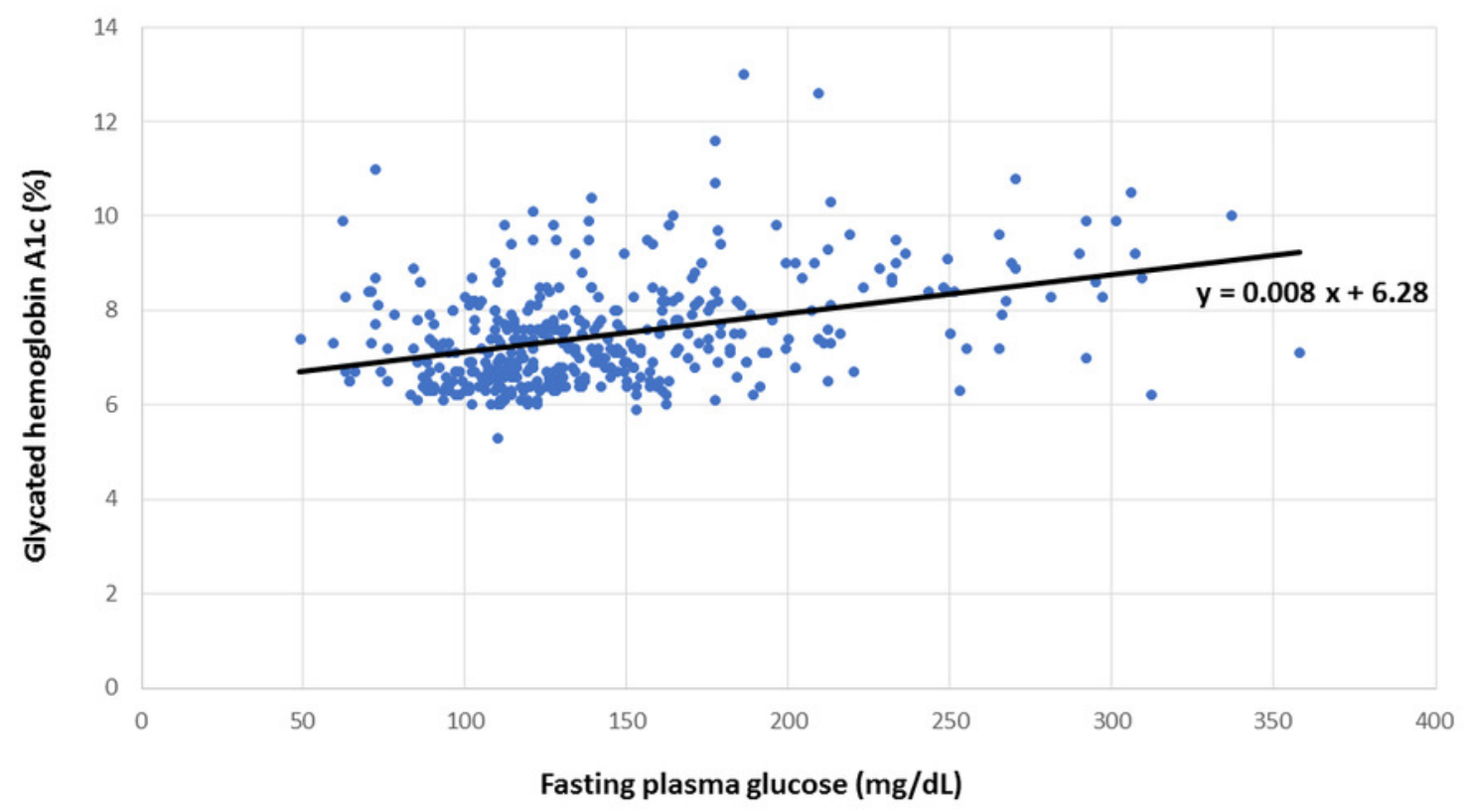


Figure 2

Enrollment protocol of the study

Enrollment protocol of the study with number and reason for candidate exclusion.

480 Individuals assessed for eligibility

57 Excluded

20 No statin prescription

32 Coronary angiography prior to study

5 No concomitant fasting glucose and hemoglobin A1c level

423 Received baseline blood test three months prior to angiography

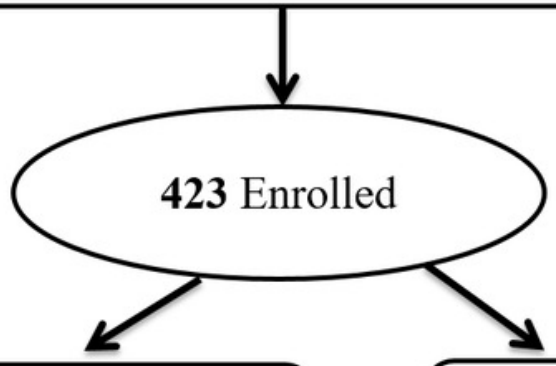

243 with hemoglobin glycation index $\geqq 0$ received coronary angiography

180 with hemoglobin glycation index $<$ 0 received coronary angiography 